\title{
Conscious error perception: The impact of response interference from a secondary task
}

\author{
Maria M. Robinson ${ }^{1}$ - David E. Irwin ${ }^{1}$
}

Published online: 31 January 2017

(C) The Psychonomic Society, Inc. 2017

\begin{abstract}
An erroneous response is not always accompanied by the conscious perception of the error being made. We examined whether increased response interference on a manual task improved the conscious perception of erroneous eye movements on a concurrent oculomotor task. In the first experiment, we examined whether a correlate of response interference, increased task difficulty alone, could improve perception of errors. We found no effect of task difficulty on selfmonitoring. Results from a second experiment suggested that participants' ability to monitor their eye movements improved with increased response interference, but post hoc analyses indicated that this was due to a decrease in corrective behaviors. Experiment 3 required participants to report directly on whether they had made an eye movement error, and we found that response interference perturbed, rather than improved, participants' ability to report on their errors. Together, these findings contribute to models of error monitoring, revealing little support for the view that general increases in response interference or task difficulty are signals that contribute to the conscious detection of errors.
\end{abstract}

Keywords Visual awareness $\cdot$ Executive control $\cdot$ Dual-task performance

The ability to detect one's own erroneous behavior is a prerequisite for adaptive behavioral adjustment. However, the detection of erroneous responses is not always accompanied by the conscious recognition of error commission. In this

Maria M. Robinson

mariamvr9@gmail.com

1 Department of Psychology, University of Illinois at UrbanaChampaign, 603 East Daniel Street, Champaign, IL 61820, USA article, we attempted to identify factors that differentiate errors that are consciously detected from those that are not.

Current models of behavior monitoring liken error detection to a decision process that is based on the accumulation of evidence over time, starting from the initial programming of an erroneous response (e.g., Rabbitt, 1968). Information that may be weighed in the evaluation of a response includes the predicted motor signal sent following the planning of a response, or efference copy. Discrepancies between an efference copy and the perceived action outcome could signal that an error has occurred (Peterburs et al., 2011).

Other researchers have proposed that response interference may be another source of evidence that contributes to the conscious detection of errors (Yeung, Botvinick, \& Cohen, 2004). According to this conflict monitoring view, a single conflict-monitoring mechanism may be sensitive to signs that cognitive control may need to be upregulated. Such a conflictmonitoring loop may be triggered by instances of response interference, which occurs when multiple motor representations are coactivated and compete with processing of goal relevant information (e.g., MacLeod, 1991; Simon, 1969). Formally, response interference is modelled as the product of two coactivated incompatible response units (weighted by the strength of the inhibitory link between them), where greater values reflect increased response conflict. An erroneous response may be viewed as an instance of response interference because, following response execution, the representation of the incorrect response competes with the representation of the correct motor plan. If a single regulatory system does not discriminate between input generated from increased response interference and input generated from erroneous responses, response interference may facilitate conscious error detection by disambiguating evidence that an error has occurred if other sources of information (e.g., goal-relevant motor representations) are degraded. Some recent studies have found support 
for shared neural correlates of processing elicited by response interference and the conscious perception of errors (for a recent review, see Klein, Ullsperger, \& Danielmeier, 2013).

Studies have focused on one neural correlate of response conflict, that is, the error-related negativity (ERN), to address the question of whether changes in ERN amplitude can predict levels of error awareness. Results from these studies have been inconsistent (for a review, see Wessel, 2012). The link between response conflict and ERN amplitude remains controversial, however, so even strong evidence for a relationship between ERN amplitude and error awareness (or lack of such a relationship) would fail to elucidate the relationship between response interference and error awareness (Steinhauser, Maier, \& Hubner, 2008).

In this set of experiments, we examined directly how general increases in response interference influence the conscious perception of errors. Previous studies examining this have relied on single-task paradigms. Instead, we used a dual-task paradigm in which we examined how error perception on one task changed as a function of different response interference levels on a second task. We reasoned that this approach directly addresses the question of whether general, non-taskspecific increases in response interference contribute to error awareness. In addition, using a dual-task design eschews several limitations of using a single task. For instance, many tasks that evoke high levels of response interference (e.g., manual flanker task) produce low rates of undetected erroneous responses. In contrast, standard tasks where the rate of undetected responses is high (e.g., antisaccade task) do not allow the researcher to manipulate response interference in a systematic fashion. To avoid the limitations of using a single task, we used a dual-task paradigm in which we coupled the antisaccade and the flanker task. Note that examining how response interference affects error awareness cross-modally also eliminates alternative explanations based on interference at the point of peripheral (response execution) rather than central (response selection) stages.

We chose the antisaccade paradigm (Hallett, 1978) because people tend to have high error rates on this task, and a large proportion of errors (as high as 50\%) are not consciously detected (Mokler \& Fischer, 1999). In traditional variants of the antisaccade task, people are instructed to fixate on a stimulus presented in the center of a computer screen. After a brief delay, a sudden onset appears to the right or left of the fixation marker. Instead of orienting toward the abrupt onset, people are instructed to shift their gaze to the opposite, mirror location of the peripheral cue. Despite these instructions, on some trials people are unable to inhibit a prepotent prosaccade to the peripheral cue (Hallett \& Adams, 1980). Furthermore, on approximately $50 \%$ of such erroneous prosaccade trials, people are unaware of their erroneous prosaccades. This contrasts with other forced-choice manual-response tasks, where conscious error perception is quite high (usually above $90 \%$ ). The high misreport rate makes the antisaccade task a prime paradigm for examining possible determinants of the conscious perception of errors.

In the flanker task, response competition is manipulated by the presentation of stimuli associated with congruent or incongruent response mappings. In this study, the flanker stimulus was a letter string in which the central letter and flanking letters were associated with a compatible or incompatible button press (Eriksen \& Eriksen, 1974). Response competition on the flanker task is reflected in slower reaction times and lower accuracy scores on response incompatible relative to response compatible trials. Neural evidence has shown that this is due to the automatic coactivation of brain regions corresponding to motor programming of both action plans on response incompatible, but not response compatible trials (DeSoto, Fabiani, Geary, \& Gratton, 2001). Successful completion of the flanker task requires the suppression of activity triggered by the flanking targets and the selective biasing of the correct, instruction-designated action plan (Nee, Wager, \& Jonides, 2007). Similar dynamics of control processing are invoked in the antisaccade task, in which people must suppress the dominant tendency to look toward a peripheral onset and generate a novel action plan to look to the side contralateral to the peripheral target. Models of antisaccade performance share the assumption that the generation of a correct antisaccade reflects a competitive process, or race, between the execution of reflexive and voluntary eye movements (Hutton \& Ettinger, 2006). The generation of correct antisaccades thus reflects higher activation of the voluntary antisaccade, and down regulation of activity of the reflexive prosaccade. The existence of competition between these two motor programs is indexed in part by the generation of fast corrective saccades, which follow erroneous prosaccades on some proportion of trials (Bowling, Hindman \& Donnelly, 2012). Such corrective saccades occur too quickly to be programmed serially after the erroneous prosaccade and thus point to parallel processing of the reflexive and voluntary eye movement.

Together, extant data support the view that the antisaccade and flanker tasks invoke similar components of an executive control network that operates in the resolution of conflict at the response selection stage of behavioral control (van Veen \& Carter, 2002). Note that we are not assuming that eye movements (in the antisaccade task) and hand movements (in the flanker task) interfere with each other during response execution, but rather that cross-task interference occurs at a more central locus. The combination of these two paradigms allows us to examine whether systematic changes of response interference on the flanker task affects conscious error detection on specific action outcomes of the antisaccade task. By presenting the flanker string at fixation, we ensured that response 
competition was evoked by the flanker stimulus at the start of each trial (Beck \& Lavie, 2005), and thus overlapped with control processing relevant to the completion of the antisaccade task. Following each trial we collected measures of conscious error monitoring by asking participants to report on the direction of their first eye movement. Of interest was whether conscious error perception of antisaccade performance would be higher when the concurrently performed flanker task generated high (response incompatible) as opposed to low (response compatible) levels of response interference.

One complication with our approach is that it is possible that the error monitoring system does not search solely for response conflict signals, but rather that increased response conflict is only one of several sources of information that contribute to error detection. According to an account given by Brown and Braver (2005), response interference may signal error likelihood because modulating conflict between competing action plans is inherently more difficult, and thus more likely to lead to error commission. A non-mutuallyexclusive view is that response interference leads to an increase in general physiological arousal and might contribute to the detection of errors through global neural changes that modulate attention networks necessary for the conscious detection of errors (Wessel, Danielmeier, \& Ullsperger, 2011; Ullsperger, Harsay, Wessel, \& Ridderinkhof, 2010). Both of these accounts predict that task difficulty, rather than response interference per se, may improve conscious error detection.

To address these possibilities, before examining the effect of response interference on error perception, we wanted to determine whether task difficulty alone improves error perception in the absence of response interference. To this end, in Experiment 1 we coupled the antisaccade task with a mental rotation task in which participants had to judge the handedness of letter stimuli (i.e., whether they were normal or mirror reversed) presented at different orientations. Previous work has shown that this is a difficult and time-consuming task because of the need to imagine the stimulus rotating to the upright before its handedness can be determined (e.g., Cooper \& Shepard, 1973). The response demands themselves are minimal, however, and thus seem unlikely to conflict with saccade execution. Accordingly, we expected that instructing participants to complete the mental rotation task and the antisaccade task simultaneously would impose high levels of mental demand (Irwin \& Carlson-Radvansky, 1996) without creating response conflict per se. Within the mental rotation task, we manipulated task difficulty by varying stimulus orientation. On the basis of previous data, we classified trials where initial stimulus orientation was $0^{\circ}, 90^{\circ}$, and $270^{\circ}$ as low-difficulty trials, and where initial stimulus orientation was $180^{\circ}$ as highdifficulty trials (Irwin \& Brockmole, 2000). If task difficulty alone influences conscious error perception, then we expected participants to be more aware of their errors on the antisaccade task on high-difficulty mental rotation trials than on lowdifficulty trials.

\section{Experiment 1}

Prominent accounts of error detection hold that conscious error detection requires the maintenance and comparison of the intended action plan and information regarding the likelihood that an erroneous response was produced or not. This type of metacognitive judgment may induce an additional memory demand (e.g., maintenance of intended action plan, and comparison of the enacted and intended action plan) that is not present if participants are simply asked to report on the action that they produced. Thus, to increase our sensitivity to possible effects of task difficulty on action monitoring, on each trial we asked participants to report on the perceived direction of their first eye movement instead of asking them to report whether they had made an error or not. Participants responded by fixating a box on the left or right side of the screen and, while fixating, pressing a button on a controller to register their eye position.

\section{Method}

Participants We conducted a power analysis using the statistical software G*Power 3.0.10 (Faul, Erdfelder, Lang, \& Buchner, 2007) to determine a minimum sample size with which we could detect a moderate effect size $\left(d_{z}=0.65\right)$ with $80 \%$ power $(\alpha=0.05)$. This analysis revealed that we would need a minimum of 32 participants. In total, 35 participants (three were excluded from analyses, as explained below) from the University of Illinois community participated in two 50minute sessions in exchange for 12 dollars. Participants reported normal or corrected-to-normal vision and were naïve as to the purpose of the experiment. The study was approved by the university's Institutional Review Board.

Stimuli and procedure Stimuli were presented on a 21-inch computer monitor (ViewSonic G810 CRT) with a refresh rate of $85 \mathrm{~Hz}$. Eye movements were recorded with an Eyelink II video-based eye tracker (SR Research Ltd., Mississauga, Ontario, Canada) with temporal resolution of $500 \mathrm{~Hz}$, spatial resolution of $0.1^{\circ}$, and pupil-size resolution of $0.1 \%$ of pupil diameter. The participants' heads were stabilized with a chin rest, fixed at $49 \mathrm{~cm}$ from the computer monitor. All stimuli were presented on a white background (luminance $=86.3 \mathrm{~cd} /$ $\mathrm{m}^{2}$ ). Participants made manual responses by pressing buttons on a Microsoft Sidewinder digital game controller interfaced with the eye-tracking computer.

Each block of trials began with a five-position calibration procedure in which the edges and center of the screen were fixated. Participants' button presses were followed by a 506- 
ms delay to reduce the effects of masking from the drift correction dot. After this delay, a letter $\left(0.8^{\circ} \times 0.8^{\circ}\right)$ and an eyemovement cue $\left(0.8^{\circ} \times 0.8^{\circ}\right.$ black cross $)$ were simultaneously presented on the computer screen. The letter was presented at the center of the screen, and the cue was presented to the left or right $\left(10^{\circ}\right.$ away from the center of the screen), and was vertically aligned with the letter. Participants were instructed to make an eye movement, as quickly as possible, to the opposite, mirror location of the cue in the periphery. To encourage fast eye movements, the letter was presented for only $200 \mathrm{~ms}$. While making their eye movement, participants were also instructed to respond, as quickly and accurately as possible, to the handedness of the letter shown in the center of the computer screen. On half of the trials, the image of the letter was normal, and on the other half of the trials, the image of the letter was mirror reversed. Within each handedness condition, the letter was rotated $0^{\circ}$ (1/6 of trials), $90^{\circ}$ ( $1 / 6$ of trials), $270^{\circ}$ ( $1 / 6$ of trials), or $180^{\circ}$ ( $1 / 2$ of trials). On the basis of previous data, we classified trials where initial stimulus orientation was $0^{\circ}, 90^{\circ}$, and $270^{\circ}$ as low-difficulty trials, and where initial stimulus orientation was $180^{\circ}$ as high-difficulty trials (Irwin $\&$ Brockmole, 2000). Trials were sequenced randomly throughout the experimental session.

Following their letter response on each trial, participants were presented with two squares, one on the left and one on the right side of the computer screen. We did not instruct participants to fixate back toward the center before the onset of the self-report screen because we were concerned that instructing participants to generate a new eye movement might override oculomotor information from the antisaccade task. Participants were asked to report on the direction of their first eye movement (i.e., left or right) by looking at the appropriate box and pressing the center button on the controller. A trial would not advance past the self-report screen unless participants fixated within an interest region defined by the area within one of the two boxes and pressed the center button. The next trial began after a 2-s intertrial interval. The experiment began with 15 practice trials that were followed by 272 experimental trials. Thus, participants completed 544 trials across the two experimental sessions. Participants were given an optional break and recalibrated after every 68th trial. An example of the sequence of events is shown in Fig. 1.

\section{Results}

For each comparison we report means, standard error of the mean (in parentheses), and the absolute value of the effect size. For comparisons that were nonsignificant, we also report a Bayes factor based on a Cauchy distribution prior scaled at $r=1.0$ (JZS BF), which quantifies evidence in favor of the null conditionalized on the observed data and sample size (Rouder, Speckman, Sun, Morey, \& Iverson, 2009).
Participant and trial exclusion criteria Three participants (out of 35) were excluded because their accuracy on the antisaccade task fell below $50 \%$ correct.

We excluded all trials on which participants' RTs on the mental rotation task fell below 200 or above $2,200 \mathrm{~ms}(8.8 \%)$, on which first saccade latencies were below 80 or above $1,200 \mathrm{~ms}(5.6 \%)$, on which participants' first fixation deviated more than $2^{\circ}$ from the central fixation presented at the start of each trial $(2.7 \%)$, on which participants failed to make a saccade $(1.0 \%)$ and on which participants made a button press before an eye movement (4.4\%). All analyses, except those of accuracy scores on the mental rotation task, excluded trials on which participants made an incorrect response on the mental rotation task (9.3\%). By these exclusion criteria, 13,501 trials were left for analysis.

Performance on the antisaccade task First, we examined whether our paradigm produced typical effects on participants' performance in the antisaccade task. We found that $7.3 \%$ of all eye movements were erroneous prosaccades. There was a significant difference between prosaccade and antisaccade latencies where prosaccade latencies (443 (35) $\mathrm{ms}$ ) were longer than antisaccade latencies (403 (30) ms), $t(29)=2.25, p=.032, \mathrm{~d}_{\mathrm{z}}=0.41$; note that the degrees of freedom are less than expected because not all subjects produced erroneous prosaccades. Mean erroneous prosaccade amplitude $\left(10.2^{\circ}(0.55)\right)$ was significantly shorter than that of correct antisaccades $\left(14.4^{\circ}\right.$ $(0.64)), t(29)=6.04, p<.001, \mathrm{~d}_{\mathrm{z}}=1.01$. We also found that people were more likely to misreport on the direction of their first eye movement when they made an erroneous prosaccade than when they made a correct antisaccade, $t(29)=5.91, p<.001, \mathrm{~d}_{\mathrm{z}}=1.08$, correctly reporting only on $50 \%$ (6) of all prosaccades and 93\% (3) of all antisaccades. Finally, the percentage of correct selfreports was significantly lower on trials in which participants made a corrective saccade back toward the antisaccade target following their erroneous prosaccade (40\% (7)) than when they did not make a corrective saccade back towards the antisaccade target $(88 \%(4)), t(18)$ $=6.57, p<.001, \mathrm{~d}_{\mathrm{z}}=1.56$. In addition, self-report accuracy was higher on prosaccade trials when participants made a slow $(50 \%(8))$ versus a fast $(<200 \mathrm{~ms})(35 \%$ (6)) corrective saccade, $t(23)=2.37, p=.027, \mathrm{~d}_{\mathrm{z}}=$ 0.48 . These results are generally consistent with those of previous antisaccade studies (e.g., Mokler \& Fischer, 1999; Taylor \& Hutton, 2011; Unsworth, Schrock, \& Engle, 2004), except that erroneous prosaccade latencies are usually faster than antisaccade latencies. The lack of such an effect in Experiment 1 was presumably due to the demanding nature of the mental rotation task, which may have caused subjects to delay saccade programming until 


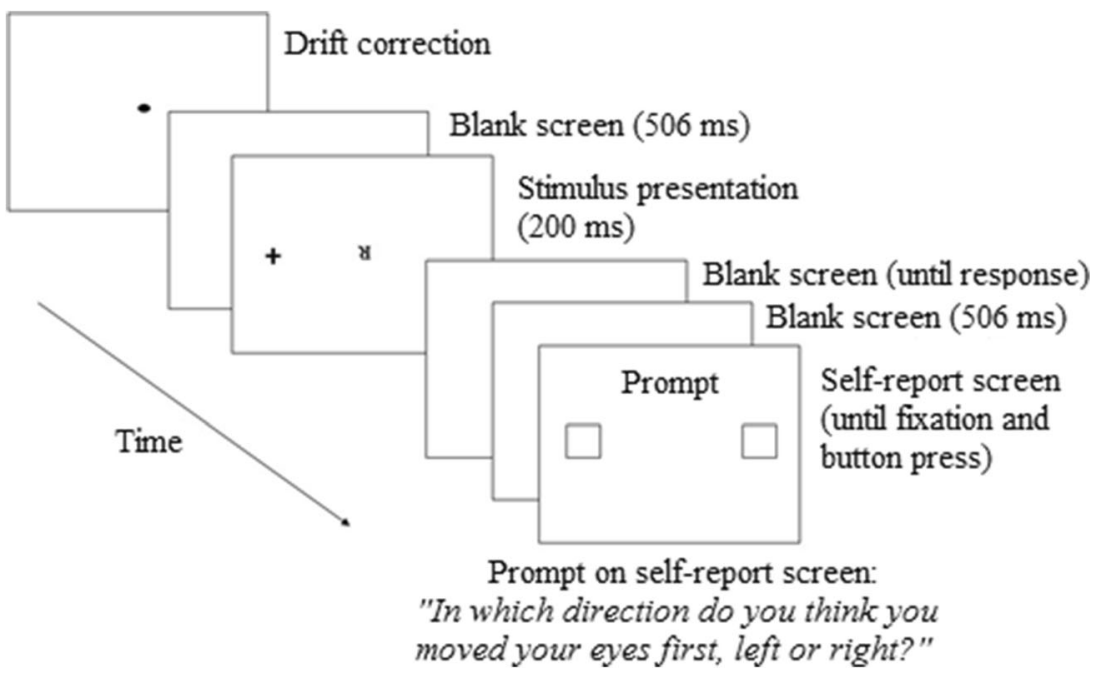

Fig. 1 Example sequence of events from Experiment 1

the letter stimulus had been identified (we elaborate on this discrepancy below).

Performance on the mental rotation task Next, we examined participants' performance on the mental rotation task. Comparisons revealed typical patterns of performance on the mental rotation task. Specifically, we found a significant effect of task difficulty on RTs, $t(31)=9.18, p<.001, \mathrm{~d}_{\mathrm{z}}=1.62$, and accuracy, $t(31)=6.77, p=.001, \mathrm{~d}_{\mathrm{z}}=1.2$, showing slower reaction times on high than on low-difficulty trials (1,168 (33) and 990 (30) ms, respectively) and lower accuracy scores on high than on low-difficulty trials (88\% (1) and 96\% (1) correct, respectively).

Effect of mental rotation on eye movements Having established that our paradigm generally produced typical patterns of performance on both interference tasks, we examined whether mental rotation condition interacted with behavioral performance on the antisaccade task. Our analyses revealed no effect of task difficulty on the programming of either the percentage of erroneous prosaccades made (low-difficulty $=7.3 \%(1.3)$, highdifficulty $=6.9 \%(1.4)), t(31)=0.5, p=.62, \mathrm{~d}_{\mathrm{z}}=0.09 ; \mathrm{JZS} \mathrm{BF}$ in favor of null hypothesis $=6.46$, or the percentage of corrective antisaccades made on erroneous prosaccade trials (low-difficulty $=73.1 \%(6.0)$, high-difficulty $=71.8 \%(6.2)), t(27)=0.34, p=$ $.734, \mathrm{~d}_{\mathrm{z}}=0.07$; JZS BF in favor of null hypothesis $=6.48$. However, we did find that antisaccade latencies were significantly shorter on low-difficulty (394 (26) ms) than on high-difficulty trials (424 (33) ms), $t(31)=3.43, p=.002, \mathrm{~d}_{\mathrm{z}}=0.61$.

Effect of mental rotation on self-report Most importantly for present purposes, we did not find a significant difference in participants' self-report accuracy (i.e., awareness of their initial saccade direction) between high and low-difficulty conditions (see Fig. 2). Participants were accurate on $51 \%$ (6) of prosaccade trials in the low-difficulty condition and on $46 \%$ (7) of prosaccade trials in the high-difficulty condition, $t(27)=0.85, p=.401, \mathrm{~d}_{\mathrm{z}}=0.16$; JZS BF in favor of null hypothesis $=4.85$.

Supplementary analyses: Effect of prosaccade latency on self-report We were concerned by the finding that participants' overall prosaccade latencies were longer than antisaccade latencies. Although this finding was unexpected we speculate that it is potentially relevant to previous evidence that attentionally demanding tasks may have opposite effects on prosaccade and antisaccade latencies. Specifically, Kristjansson, Chen, and Nakayama (2001) found that a difficult visual attention (discrimination) task slowed prosaccade latencies, while also increasing the speed of antisaccade latencies. The authors argued that prosaccades are not attentionless eye movements (a view consistent with other evidence; Godijn \& Kramer, 2006), but rather that the capture of attention requires attentional resources. Accordingly, taxing attention at the time at which people are presented with a sudden onset may increase saccade latencies toward that onset because attentional resources are not available

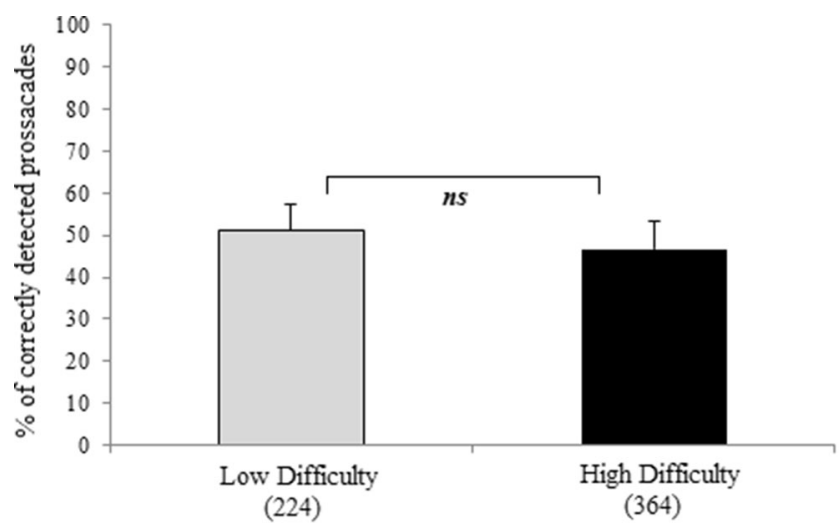

Fig. 2 Percentage of correctly detected prosaccades as a function of mental rotation condition. Average number of trials per cell in each condition is reported in parentheses 
for its processing. Furthermore, requiring participants to make a voluntary antisaccade shortly after taxing attention may decrease antisaccade latencies because participants are no longer required to inhibit the prepotent prosaccade eye movement. Although these authors did not find that prosaccade latencies were longer than antisaccade latencies (rather prosaccade latencies increased relative to a baseline condition and antisaccade latencies decreased relative to a baseline condition), it is likely that our mental rotation task was substantially more difficult than the discrimination task used by Kristjansson et al., and thus may have accentuated the reversed difference between prosaccade and antisaccade latencies. This interpretation is not entirely consistent with our finding that antisaccade latencies increased (rather than decreased) as a function of difficulty condition. We also found no effect of difficulty condition on prosaccade latencies, $F(1,29)=$ $0.15, p=.812$, whereas one might expect that prosaccade latencies would get longer with increasing load. However, it is possible that this effect on attention reflects a general dual-task cost, rather than the effects of mental rotation difficulty per se.

Regardless of the cause for this difference in latencies between prosaccades and antisaccades, it is possible that the additional load imposed by the mental rotation task leads to a fundamentally different type of erroneous response than what is traditionally observed on the antisaccade task. Although this is not necessarily problematic in the context of our research goal, which is to examine whether task difficulty affects the detection of any type of error, we wanted to examine more systematically whether self-report accuracy differed as a function of difficulty under conditions where performance is more like what is typically observed in the antisaccade task. To this end, we used a median split analysis (based on median prosaccade latencies calculated separately for each participant) to compare error awareness between trials where participants made relatively fast ( $<$ median) or relatively slow $(\geq$ median) erroneous prosaccades. We found that prosaccade latencies of the fastest (<median) portion of prosaccades (292 (24) ms) were substantially faster than those of antisaccades (389 (33) $\mathrm{ms}), t(27)=4.84, p<.001, \mathrm{~d}_{\mathrm{z}}=0.91$. Thus, as we intended, this analysis helped isolate those prosaccades that are traditionally observed in the antisaccade task. An ANOVA with mental rotation condition and prosaccade latency (fast versus slow, based on median split analysis) as the two within-subjects factors and self-report accuracy on prosaccade trials as the dependent variable, revealed no effect of prosaccade latency, $F(1,27)=0.001, p=.984$, no effect of mental rotation condition, $F(1,27)=0.612, p=.441$, and most importantly, no interaction between prosaccade latency and mental rotation condition, $F(1,27)=0.62, p=.437$, on error awareness. Self-report accuracy was $54.9 \%$ (7) and $53.1 \%$ (7) correct when prosaccades were fast - that is, below individual median, in the low and high difficulty conditions, respectively, and $57.1 \%$ (6) and $51 \%$ (7) correct when prosaccades were slow - that is, equal to or above individual median, in the low and high difficulty conditions, respectively. In sum, these supplementary analyses suggest that the lack of effect of difficulty on self-report is likely not due to the fact that completing the mental rotation task led participants to commit fundamentally different types of errors on the antisaccade task.

\section{Discussion}

In sum, Experiment 1 revealed no support for the view that increased task difficulty affects conscious error perception. Some authors have proposed that response interference may improve error detection indirectly because it makes a task more difficult and thus signals increased likelihood of error commission. According to this account, it is difficulty that contributes to the conscious perception of errors rather than response interference per se. Increasing the difficulty of the mental rotation task interfered with antisaccade performance by increasing antisaccade latencies, but had no effect on subjects' awareness of their eye movements. Thus, our data suggest that in the absence of response interference, task difficulty by itself may not improve error detection.

\section{Experiment 2}

Having found no evidence that increased task difficulty alone improves error detection, we turned to our primary research question, that is, whether response interference increases error awareness. To address this question we modified the design from Experiment 1 by replacing the mental rotation task with the flanker task.

\section{Method}

Participants Thirty-six participants (four were excluded from analyses, as explained below) from the University of Illinois participated in two 50-minute sessions in exchange for 12 dollars. Participants reported normal or corrected-to-normal vision and were naïve as to the purpose of the experiment. None had participated in Experiment 1.

Stimuli and procedure The apparatus was the same as in Experiment 1. Each block of trials began with a fiveposition calibration procedure in which the edges and center of the screen were fixated. Following the first calibration, participants completed a training sequence of 160 trials to become familiar with the stimulus-response mappings that would be used in the experimental phase of the study. During this training sequence, participants were instructed to press specified triggers when presented with certain letters in the center of the computer screen: When shown the letters $S$ or $M$, participants pressed the right trigger with the index finger of their right hand; when presented with the letters $P$ or $H$, 
participants pressed the left trigger with the index finger of their left hand. Participants were instructed to respond as quickly and accurately as possible on each trial. Trials did not advance until participants made a correct response and participants were given auditory feedback for every incorrect trigger press. The auditory feedback was a low tone lasting $1 \mathrm{~s}$.

During the experimental phase of the study (Fig. 3), participants began each trial by fixating on a drift-correction point at the center of the screen and pressing the center button of the controller. Participants' button presses were followed by a 506-ms delay to reduce the effects of masking from the drift correction dot. After this delay, five letters (size of letter string: $\left.3.7^{\circ} \times 0.8^{\circ}\right)$ and an eye-movement cue $\left(0.8^{\circ} \times 0.8^{\circ}\right.$ black cross) were simultaneously presented on the computer screen. The letter string was presented at the center of the screen and the cue was presented to the left or right $\left(10^{\circ}\right.$ away from the center of the screen) and was vertically aligned with the letter string. Participants were instructed to make an eye movement, as quickly as possible, to the opposite, mirror location of the cue in the periphery. To encourage fast eye movements, the central letter string was presented for only $200 \mathrm{~ms}$. While making their eye movement, participants were also instructed to respond, as quickly and accurately as possible, to the center (target) letter of the five-letter string. Participants were instructed to ignore the two flanking letters presented on each side of the target letter. The flankers consisted of identical letters. Target and flanker letters could range in similarity, reflecting one of three possible flanker interference conditions, as explained next. On No Interference (NI) trials, the flanking letters matched the target letter (e.g., SSSSS); on Perceptual Interference (PI) trials, the flanking letters were perceptually different from the target letter but were mapped onto the same response (e.g., SSMSS); and on Response Interference (RI) trials, the target and flanker letters were mapped onto incongruent responses (e.g., SSPSS).
Both experimental sessions included 160 training trials, 15 practice trials, and 272 experimental trials (totaling 544 experimental trials for each participant across the two experimental sessions). Participants were given an optional break, and recalibrated after every 68th trial. Out of the 272 experimental trials, 50\% were No Interference, $25 \%$ were Perceptual Interference, and $25 \%$ were Response Interference trials. These proportions are based on previous literature showing that the amount of conflict due to stimulus and response interference is modulated by the overall context of the experimental task, and that a relatively greater proportion of congruent trials increases the effects of both stimulus and response interference (e.g., Lindsay \& Jacoby, 1994; Logan \& Zbrodoff, 1979). During the experimental phase, the antisaccade cue was presented equally often on each side (left or right) of the computer screen. The type of flanker condition and antisaccade cue location were sequenced randomly across trials. Following their response on each trial, participants were presented with two squares, one on the left and one on the right side of the computer screen. Participants were asked to report on the direction of their first eye movement (i.e., left or right) by looking at the appropriate box and pressing the center button on the controller. A trial would not advance past the self-report screen unless participants fixated within an interest region defined by the area within one of the two boxes and pressed the center button. The next trial began after a 2 -s intertrial interval.

\section{Results}

Participant and trial exclusion criteria Three participants did not show up for the second experimental session and were excluded from analyses. A fourth participant was excluded because he or she made antisaccades on fewer than $50 \%$ of trials.

We excluded all trials on which participants' RTs fell below 200 or above $2,200 \mathrm{~ms}(2.1 \%)$, on which first saccade

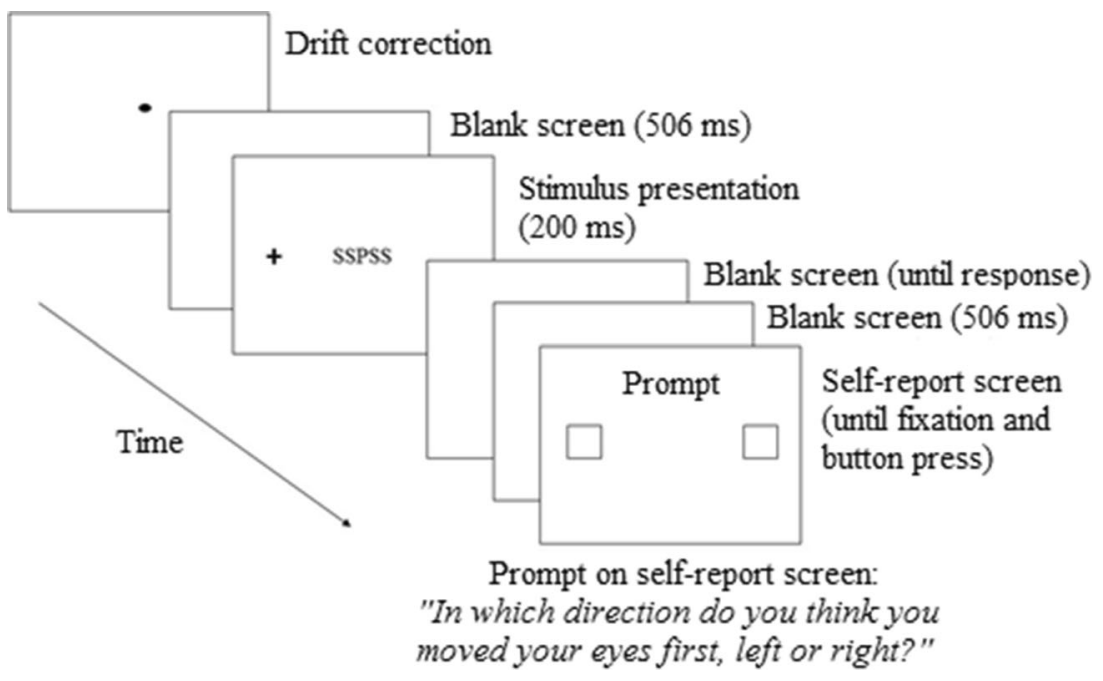

Fig. 3 Example sequence of events in Experiment 2 
latencies were below 80 or above $1,200 \mathrm{~ms}(1.8 \%)$, on which participants' first fixation deviated more than $2^{\circ}$ from the central fixation presented at the start of each trial $(3.4 \%)$, on which participants failed to make a saccade $(0.1 \%)$ and on which participants made a button press before an eye movement (2\%). All analyses, except those of accuracy scores on the flanker task, excluded trials on which participants made an incorrect response on the flanker task (4\%). By these exclusion criteria, 15,504 trials were left for analysis.

Performance on the antisaccade task First, we examined whether our paradigm produced typical effects on participants' performance in the antisaccade task. We found that $5.2 \%$ of all eye movements were erroneous prosaccades. The mean latency of erroneous prosaccades (306 (17) $\mathrm{ms}$ ) was shorter than that of correct antisaccades (325 (14) ms), $t(31)=2.05, p=.048, \mathrm{~d}_{\mathrm{z}}=$ 0.36 . Mean erroneous prosaccade amplitude $\left(10.5^{\circ}(0.5)\right)$ was significantly shorter than that of correct antisaccades $\left(13.3^{\circ}\right.$ $(0.5)), t(31)=8.3, p<.001, d_{z}=1.47$. We also found that people were more likely to misreport on the direction of their first eye movement when they made an erroneous prosaccade than when they made a correct antisaccade, $t(31)=5.77, p<.001, \mathrm{~d}_{\mathrm{z}}=1.02$, correctly reporting only on $65 \%$ (5) of all prosaccades and $95 \%$ (1) of all antisaccades. The percentage of correct self-reports was significantly lower on trials in which participants made a corrective saccade back toward the antisaccade target following their erroneous prosaccade (49\% (6)) than when they did not make a corrective saccade back towards the antisaccade target $(99 \%$ $(0.2)),\left(t(29)=8.91, p<.001, d_{z}=1.63\right)$. Furthermore, self-report accuracy on prosaccade trials was lower when participants made a fast corrective saccade $(<200 \mathrm{~ms})(54 \%$ (7)) than when they made a slow corrective saccade (79\% (5)), $t(27)=4.20, p<$ $.001, \mathrm{~d}_{\mathrm{z}}=0.78$. All of these findings are consistent with other studies that have examined conscious awareness of performance in the antisaccade task (e.g., Mokler \& Fischer, 1999).

Performance on the flanker task Next, we examined participants' performance on the flanker task. A repeated-subjects ANOVA, with flanker condition as the single factor, revealed a significant effect of factor compatibility, $F(2,62)=10.4, p<$ .001 . We found no significant difference in accuracy between NI (97\% (1)) and PI (97\% (1)) trials, $t(31)=1.87, p=.072, \mathrm{~d}_{\mathrm{z}}$ $=0.33$; JZS BF in favor of null hypothesis $=1.45$, but a significant difference in accuracy between NI (97\% (1)) and RI $(95 \%$ (1)) trials, $t(31)=3.17, p=.004, \mathrm{~d}_{\mathrm{z}}=0.56$, and PI (97\% (1)) and RI (95\% (1)) trials, $t(31)=3.59, p=.001, \mathrm{~d}_{\mathrm{z}}=0.63$. We also found a significant effect of flanker compatibility on RTs, $F(2$, $62)=30.8, p<.001$. RTs were slightly slower on NI (827 (38)) than PI trials $(815(36)), t(31)=2.30, p=.028, \mathrm{~d}_{\mathrm{z}}=0.41$. Most important, RTs were slower on RI (865 (37)) than on NI (827(38)) trials, $t(31)=4.89, p<.001, \mathrm{~d}_{\mathrm{z}}=0.86$, and on RI (865 (37)) than on PI $(815$ (36)) trials, $t(31)=7.34, p<.001$, $\mathrm{dz}=1.30$. This pattern of results replicates standard response congruency effects on the flanker task (Eriksen \& Eriksen, 1974).

Effect of flanker compatibility on eye movements Having established that our paradigm produced typical patterns of performance on both interference tasks, we examined whether flanker condition interacted with behavioral performance on the antisaccade task. We did not make predictions about the direction of this effect because we supposed that if cross-task interactions existed, performance on the antisaccade task could be either hurt by increased response interference on the flanker task (e.g., because both tasks recruit a single resource), or improved by increased response interference on the flanker task (e.g., due to upregulation of controlled processing). Once again, a single-factor within-subjects ANOVA revealed a significant effect of flanker condition on the proportion of erroneous prosaccades made, $F(2$, $62)=12.3, p<.001$. The results are shown in Fig. 4. Because these analyses were not confirmatory, we used Bonferronicorrected $t$ tests (critical $p$ value $=.016$ ). These revealed no statistically significant difference between NI $(94 \%$ (1)) and PI $\left(96 \%\right.$ (1)) trials, $t(31)=2.44, p=.021, \mathrm{~d}_{\mathrm{z}}=0.43$. Furthermore, we found that participants made significantly fewer antisaccades on RI (93\% (1)) than on NI (94\% (1)), $t(31)=2.83, p=.008, \mathrm{~d}_{\mathrm{z}}=$ 0.50 , and on RI $(93 \%$ (1)) than on PI $(96 \%$ (1)) trials, $t(31)=$ $4.38, p=.001, \mathrm{~d}_{\mathrm{z}}=0.77$. Together, these results indicate that increased response interference on the flanker task interferes with response selection on the antisaccade task. The finding that participants made fewer antisaccades on NI than on PI trials is somewhat unexpected in this context. However, this difference was not significant with a correction for multiple comparisons in this exploratory set of analyses. Furthermore, in Experiment 3, we replicated the effect of RI on the proportion of correct antisaccades, but not the difference in antisaccades between NI and PI trials. We found that the proportion of corrective saccades made also differed as a function of flanker condition, $F(2,50)=$

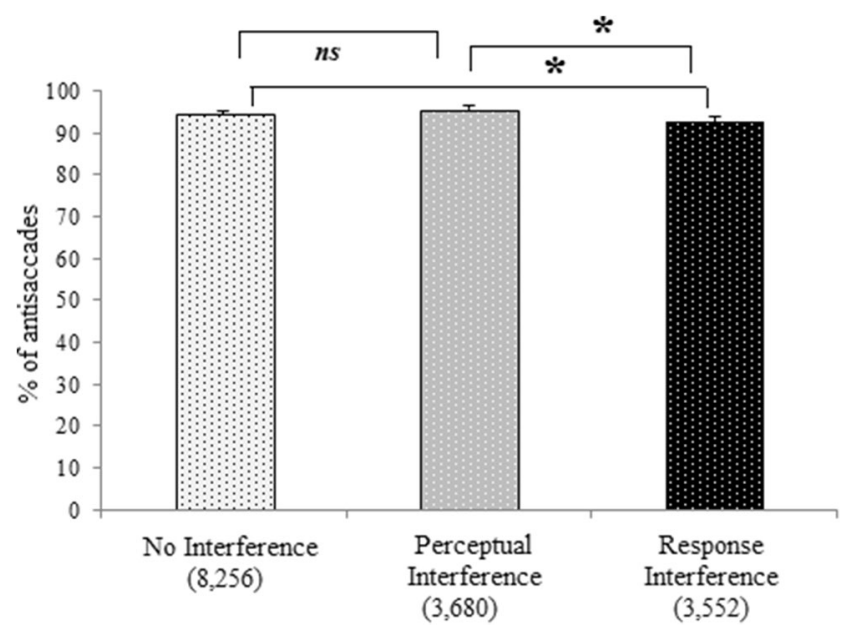

Fig. 4 Percentage of antisaccades as a function of flanker condition $\left(^{*}=\right.$ significant difference between conditions). Average number of trials per cell in each condition is reported in parentheses 
$7.25, p=.002$. Pairwise comparisons revealed a significant difference in the proportion of corrective antisaccades made between NI (78\% (5)) and RI (61\% (6)) trials, $t(25)=3.03 p=$ $.006, \mathrm{~d}_{\mathrm{z}}=0.59$, and between PI and RI trials, $t(25)=2.8, p=$ $.01, \mathrm{~d}_{\mathrm{z}}=0.55$, but not between NI $(78 \%(5))$ and PI $(80 \%(6))$ trials, $t(25)=0.73, p=0.47, \mathrm{~d}_{\mathrm{z}}=0.14$; JZS BF in favor of null hypothesis $=5.12$. We also found an effect of flanker condition on antisaccade latencies, $F(2,62)=5.85, p=.005$. Antisaccade latencies were faster on NI (322 (13) ms) than on RI (330 (15) ms) trials, $t(31)=2.83, p=.008, \mathrm{~d}_{\mathrm{z}}=0.50$, and marginally faster on PI (324 (14) ms) than on RI (330 (15) ms) trials, $t(31)=2.53, p=.017, \mathrm{~d}_{\mathrm{z}}=0.45$. Antisaccade latencies were not different between NI (322 (13) ms) and PI (324 (14) ms) trials, $t(31)=1.03, p=.313, \mathrm{~d}_{\mathrm{z}}=0.18 ; \mathrm{JZS} \mathrm{BF}$ in favor of null hypothesis $=4.39$.

Effect of flanker compatibility on self-report Having found cross-task interactions at the behavioral level (confirming that we successfully increased cross-task response interference) we examined the effects of flanker condition on awareness of errors in the antisaccade task. An ANOVA revealed a significant effect of flanker condition on participants' awareness of errors. Follow-up $t$ tests revealed no difference in participants' awareness of errors in the antisaccade task between flanker NI $(63 \%$ (7)) and PI (56\% (7)) trials, $t(25)=1.39, p=.177, \mathrm{~d}_{\mathrm{z}}=0.27$; JZS BF in favor of null hypothesis $=2.68$, but a significant difference between PI (56\% (7)) and RI (71\% (5)) trials, $t(25)$ $=2.17, p=.04, \mathrm{~d}_{\mathrm{z}}=0.43$. Furthermore, although the difference between NI $(63 \%(7))$ and RI (71\% (5)) flanker trials was not significant, $t(25)=1.46, p=.155, \mathrm{~d}_{\mathrm{z}}=0.28$; JZS BF in favor of null hypothesis $=2.45$, the data trended in a direction consistent with the prediction that response interference may improve error awareness. Together, this pattern of results provides tentative support for the view that response interference may improve error awareness (see Fig. 5).

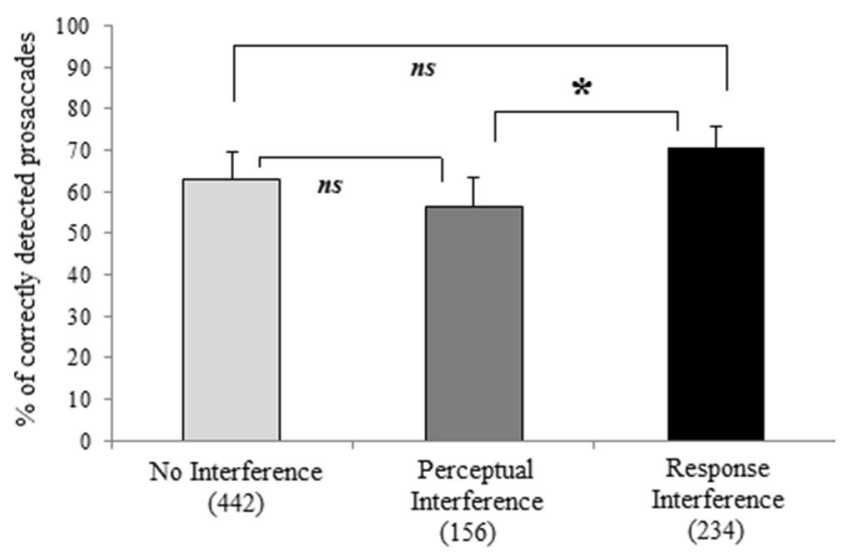

Fig. 5 Percentage of correctly detected erroneous prosaccades as a function of flanker condition $(*=$ statistically significant difference between conditions). Average number of trials per cell in each condition is reported in parentheses
Effect of corrected errors on self-report We were concerned that the impact of response interference on the self-report of eye-movement behavior might have been indirect. In particular, it seemed possible that participants were "better" at detecting erroneous prosaccades on RI trials because they made fewer corrective eye movements on such trials. A failure to program and enact a corrective saccade may have improved discriminability of erroneous prosaccades because participants were less likely to confuse representations of an intended correct response and an incorrect response during action evaluation (Blakemore $\&$ Frith, 2003). Furthermore, recall that the way we assessed self-report accuracy was by having subjects fixate a box on the side of the screen toward which they had directed their initial saccade. Because subjects were less likely to make a corrective eye movement on RI trials, their self-report accuracy on RI trials may have been inflated because they would be fixating the location where they had first moved their eyes when the self-report screen appeared. In contrast, on trials in which a corrective antisaccade was made (which was more likely to be on NI and PI rather than on RI trials), subjects would have to saccade back to the opposite side of the display to fixate the self-report screen corresponding to the location of their initial saccade, and if they failed to do this, their self-report would be judged to be in error. In other words, in some sense the finding that self-report of erroneous prosaccades was higher on RI than on NI and PI trials may have been a consequence of the way that we measured self-reports.

To address this concern, we conducted additional supplementary analyses to test whether the effects of selfreport were qualified by participants' tendency to make a greater proportion of corrective antisaccades on NI and PI than on RI trials. To this end, we compared self-report accuracy between flanker conditions separately for trials in which participants did make a corrective antisaccade, and for trials in which they did not make a corrective antisaccade. We reasoned that, if response interference has a direct effect on conscious error perception, we should see high self-report accuracy both on trials in which participants made a corrective antisaccade and on trials in which they did not make a corrective antisaccade. Instead, we found that on trials when participants did not make a corrective antisaccade there was no, $F(2,46)=$ $0.410, p=.666$, effect of flanker condition on self-report accuracy. Furthermore, we found no difference in selfreport accuracy on trials when participants did make a corrective saccade after making an erroneous prosaccade, $(F(2,16)=0.367, p=0.698$ (see Fig. 6). Levels of accuracy on trials when participants did not make a corrective antisaccade suggest that self-report accuracy is at ceiling in all three flanker conditions, most likely because 


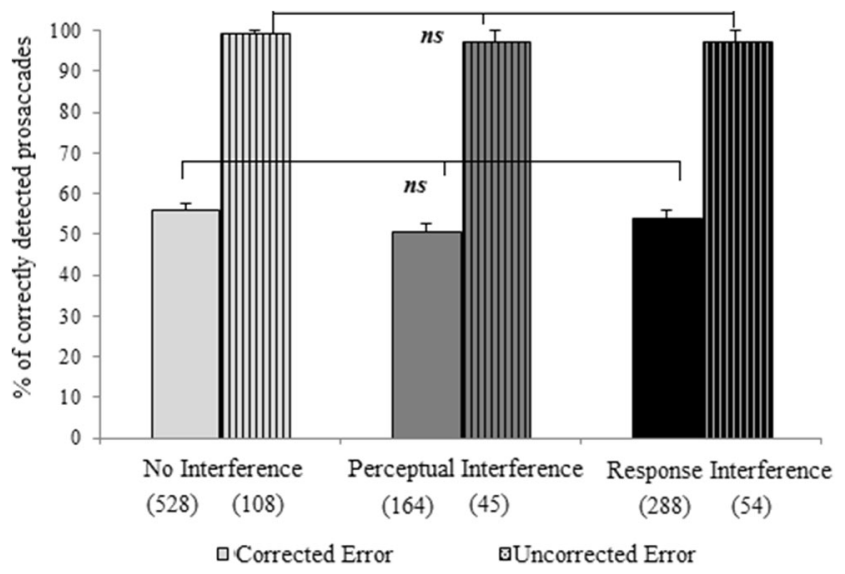

Fig. 6 Percentage of detected prosaccades as a function of whether participants made a corrective saccade and flanker condition. Average number of trials per cell in each condition is reported in parentheses

participants continue to maintain fixation at the initial saccade position until the onset of the self-report screen. Because participants made fewer corrective antisaccades on RI than on NI and PI trials, participants' overall self-report accuracy was thus inflated in the RI condition relative to the NI and PI condition. Together, these results indicate that differences in self-report of eye-movement behavior were likely not directly driven by increased response interference, but rather by a reduction in participants' tendency to produce corrective responses on RI trials.

\section{Discussion}

Results from Experiment 2 revealed an influence of response interference in the flanker task on the generation of eye movements in the antisaccade task. That is, we found that participants made fewer voluntary antisaccades and fewer corrective antisaccades on trials in which they had to produce a manual response to a response incompatible flanker stimulus. This indicates that we successfully increased response interference using our dual-task paradigm. In addition, we found that participants' self-report accuracy was higher when they had made an erroneous eye movement in the RI than in the NI and PI conditions. Follow-up analyses indicated, however, that response interference affected self-report indirectly by decreasing the frequency of corrective antisaccades on RI trials. Specifically, participants were less likely to make a corrective antisaccade on RI trials and instead maintained fixation at the location where they had first moved their eyes until the onset of the self-report screen, thereby inflating self-report accuracy on RI trials. Supplementary analyses that examined self-report accuracy conditionalized on whether a corrective antisaccade was made or not showed no differences between RI and NI or PI trials, indicating that response interference may have affected self-report accuracy only indirectly. We examined this possibility further in Experiment 3.

\section{Experiment 3}

Our findings from Experiment 2 suggest that instead of introspecting on their errors on some portion of trials, participants tended to simply remain fixated at the location where they looked last. This strategy offloads the requirements of having to keep in memory the spatial direction of their first eye movement and compare it to the direction of their intended eye movement. That is, if participants did not make a corrective eye movement after making an erroneous prosaccade, then they could simply leave their eyes where they landed initially to make their self-report instead of having to evaluate whether their eyes had moved in the intended direction. The aim of Experiment 3 was to eliminate this strategy by requiring subjects to engage error-detection processes of memory and comparison. To this end, we applied the design from Experiment 2, but changed the nature of the selfreport. Instead of having subjects fixate on the side of the screen toward which they had made their initial saccade, we instructed participants to report, by pressing a response button, on whether they thought they had made an erroneous eye movement or not. Specifically, on a trial-by-trial basis, participants were instructed to press one button if they thought they made an erroneous prosaccade and a different button if they thought they did not make an erroneous prosaccade. It is worth noting that this manipulation introduces two differences between the procedure used in Experiment 2. First, participants no longer reported with an eye movement, and therefore had to explicitly assess, or recall, the position of their first eye movement to complete the self-report portion of the task. Second, participants could no longer report on the spatial direction (left or right) where they had made their first eye movement. Thus, participants could no longer base their judgment solely on their eye position; instead, they had to maintain the representation of their initial eye movement and evaluate it against the intended eye movement (i.e., antisaccade).

\section{Method}

Participants Thirty-six participants (four were excluded from analyses, as explained below) from the University of Illinois participated in two 50-minute sessions in exchange for 12 dollars. Participants reported normal or corrected-to-normal vision and were naïve as to the purpose of the experiment. None had participated in either of the first two experiments.

Procedure Experiment 3 was identical to Experiment 2 except participants were instructed to report on error awareness by pressing one button on a hand-held response device if they thought they made an erroneous eye movement (i.e., prosaccade) and a different button if they thought they correctly made an antisaccade. 


\section{Results}

Participant and trial exclusion criteria Four participants did not show up for the second experimental session and were replaced, yielding a final sample size of 32 participants.

As before, we excluded trials on which participants' RTs fell below 200 or above 2,200 ms (3.7\%), on which first saccade latencies were below 80 or above $1,200 \mathrm{~ms}(2.8 \%)$, on which participants' first fixation deviated more than $2^{\circ}$ from the central fixation presented at the start of each trial $(2.1 \%)$, on which participants failed to make a saccade (1.3\%) and on which participants made a button press before an eye movement (1.9\%). All analyses, except those of accuracy scores on the flanker task, excluded trials on which participants made an incorrect response on the flanker task (3.4\%). By these exclusion criteria, 15,026 trials were left for analysis.

Performance on the antisaccade task We found that $5.6 \%$ of all eye movements were erroneous prosaccades. There was a marginally significant difference between mean latencies of erroneous prosaccades (281 (16) ms) and correct antisaccades (298 (13) ms), $t(31)=1.99, p=.056, \mathrm{~d}_{\mathrm{z}}=0.35$, and, as before, mean amplitude of erroneous prosaccades $\left(10.5^{\circ}(0.5)\right)$ was significantly shorter than that of correct antisaccades $\left(13.7^{\circ}\right.$ (14)), $t(31)=6.93, p<.001, \mathrm{~d}_{\mathrm{z}}=1.22$. We also found that people were more likely to misreport on the direction of their first eye movement when they made an erroneous prosaccade than when they made a correct antisaccade, $t(31)=10.55, p<$ $.001, \mathrm{~d}_{\mathrm{z}}=1.86$, correctly reporting only on $42 \%$ (5) of all prosaccades and $96 \%$ (1) of all antisaccades. All of these findings are consistent with other studies that have examined conscious awareness of performance in the antisaccade task (e.g., Mokler \& Fischer, 1999). Finally, unlike in Experiment 2, correct self-report rates were significantly lower on trials in which participants did not make a corrective saccade back toward the antisaccade target following their erroneous prosaccade $(29 \%(8))$ than when they did make a corrective saccade back towards the antisaccade target $(48 \%(6)), t(25)=$ $2.73, p=.012, \mathrm{~d}_{\mathrm{z}}=0.54$. Also unlike Experiment 2, participants were not significantly better at reporting on their erroneous eye movements when they made a slow $(66 \%(6))$ versus a fast $(<200 \mathrm{~ms})(57 \%(7))$ corrective saccade, $t(27)=$ $1.15, p=.258, \mathrm{~d}_{\mathrm{z}}=0.22$; JZS BF in favor of null hypothesis $=3.66$.

Performance on the flanker task Next, we examined participants' performance on the flanker task. We found an effect of flanker condition on accuracy, $F(2,62)=14.2, p<.001$, exhibited by a standard response compatibility effect, where accuracy was worse on RI (93\% (1)) than on NI (95\% (1)) trials, $t(31)=3.61, p=.001, \mathrm{~d}_{\mathrm{z}}=0.64$, and lower on RI $(93 \%$ (1)) than on PI $\left(96 \%\right.$ (1)) trials, $t(31)=4.24, p<.001, \mathrm{~d}_{\mathrm{z}}=0.75$. Furthermore, we found that accuracy was slightly worse on NI
$(95 \%(1))$ than on PI $(96 \%(1))$ trials, $t(31)=2.11, p=.043$, $\mathrm{d}_{\mathrm{z}}=0.37$. Once again, we also found a significant effect of flanker condition on RTs, $F(2,62)=8.36, p<.001$. RTs were significantly lower on NI (870 (47) ms) than on PI (889 (49) ms) trials, $t(31)=3.09, p=.004, \mathrm{~d}_{\mathrm{z}}=0.55$, and on NI than on RI trials, $t(31)=3.84, p<.001, \mathrm{~d}_{\mathrm{z}}=0.68$. The difference between PI (889 (49) ms) and RI (904 (46) ms) trials was trending in the direction consistent with a response compatibility effect, that is, with RTs being slower on RI than on PI trials, $t(31)=1.56, p=.128, \mathrm{~d}_{\mathrm{z}}=0.28$; JZS BF in favor of null hypothesis $=2.33$.

Effect of flanker compatibility on eye movements We found a significant effect of flanker condition on the proportion of antisaccades made, $F(2,62)=13.4, p<.001$. The results are shown in Fig. 7. Replicating the effects of flanker response congruency on eye movement programming that we found in Experiment 2, participants made significantly fewer antisaccades on RI (93\% (1)) than on NI (95\% (1)) trials, $t(31)=4.58, p<.001, \mathrm{~d}_{\mathrm{z}}=0.81$, and fewer antisaccades on RI $(93 \%$ (1)) than on PI $(95 \%$ (1)) trials, $t(31)=4.18, p<.001$, $\mathrm{d}_{\mathrm{z}}=0.74$. There was no difference in the proportion of antisaccades made between NI (95\% (1)) and PI (95\% (1)) trials, $t(31)=0.11, p=.913, \mathrm{~d}_{\mathrm{z}}=0.02 ; \mathrm{JZS} \mathrm{BF}$ in favor of null hypothesis $=7.26$. Once again, we found an effect of flanker condition on the proportion of corrective antisaccades made, $F(2,50)=5.55, p<.001$. Participants made fewer corrective antisaccades on PI (79\% (6)) than on NI $(89 \%$ (4)) trials, $t(25)=2.12, p=.044, \mathrm{~d}_{\mathrm{z}}=0.4$, and on NI $(89 \%$ (4)) than on RI (70\% (4)) trials, $t(25)=4.36, p<.001, \mathrm{~d}_{\mathrm{z}}=$ 0.84 . The difference between PI (79\% (6)) and RI (70\% (4)) trials did not reach significance, $t(25)=1.33, p=.2, \mathrm{~d}_{\mathrm{z}}=0.26$; JZS BF in favor of null hypothesis $=2.89$, but numerically the data were consistent with an effect of response congruency on

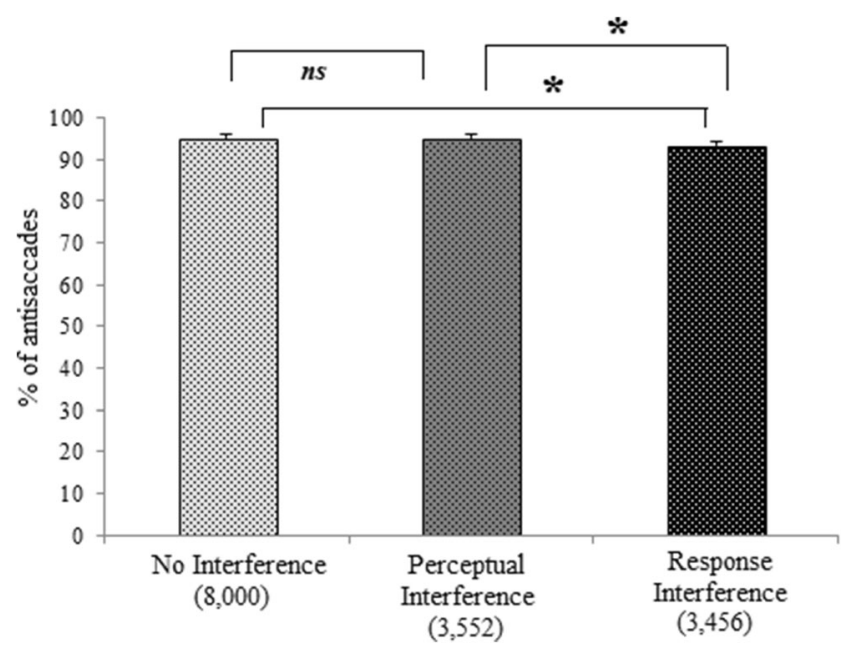

Fig. 7 Percentage of antisaccades as a function of flanker condition $\left(^{*}=\right.$ statistically significant difference between conditions). Average number of trials per cell in each condition is reported in parentheses 
corrective saccades. Finally, we found an effect of flanker condition on antisaccade latencies, $F(2,62)=7.19, p<.001$. Antisaccade latencies were faster on NI $(295(13) \mathrm{ms})$ than on PI (299 (14) ms) trials, $t(31)=3.00, p=.005, \mathrm{~d}_{\mathrm{z}}=0.53$, and faster on NI (295 (13) ms) than RI (302 (14) ms) trials, $t(31)=$ $3.27, p=.003, \mathrm{~d}_{\mathrm{z}}=0.58$. The difference between PI (295 (13) $\mathrm{ms})$ and RI (302 (14) ms) trials was not significant, but trended in the direction consistent with an effect of response congruency, $t(31)=1.33, p=.194, \mathrm{~d}_{\mathrm{z}}=0.24 ; \mathrm{JZS}$ BF in favor of null hypothesis $=3.15$.

Effect of flanker compatibility on self-report We found an effect of flanker condition on the percentage of accurate selfreports on trials in which participants made an erroneous prosaccade, $F(2,50)=5.84, p=.005$; however, unlike in Experiment 2, participants were worse at detecting erroneous eye movements on RI (34\% (6)) than on NI (47\% (6)) trials, $t(25)=2.77, p=.01, \mathrm{~d}_{\mathrm{z}}=0.54$, and worse at detecting erroneous eye movements on RI (34\% (6)) than on PI (53\% (8)) trials, $t(25)=3.5, p<.002, \mathrm{~d}_{\mathrm{z}}=0.68$. The results are shown in Fig. 8. There was no difference in the awareness of errors between NI (47\% (6)) and PI (53\% (8)) trials, $t(25)=0.82, p$ $=.418, \mathrm{~d}_{\mathrm{z}}=0.16 ; \mathrm{JZS} \mathrm{BF}$ in favor of null hypothesis $=4.8$.

\section{Discussion}

In Experiment 3, we replicated the effect of response interference on eye movement programming that we found in Experiment 2. Specifically, we found that participants made more erroneous prosaccades on trials when the flanker stimulus evoked response interference than on trials when it did not. Most importantly, we found an effect of response interference on participants' ability to detect errors, but this time we found that increased response interference perturbed participants' ability to detect erroneous responses. These findings suggest that the benefit of response interference found in Experiment 2

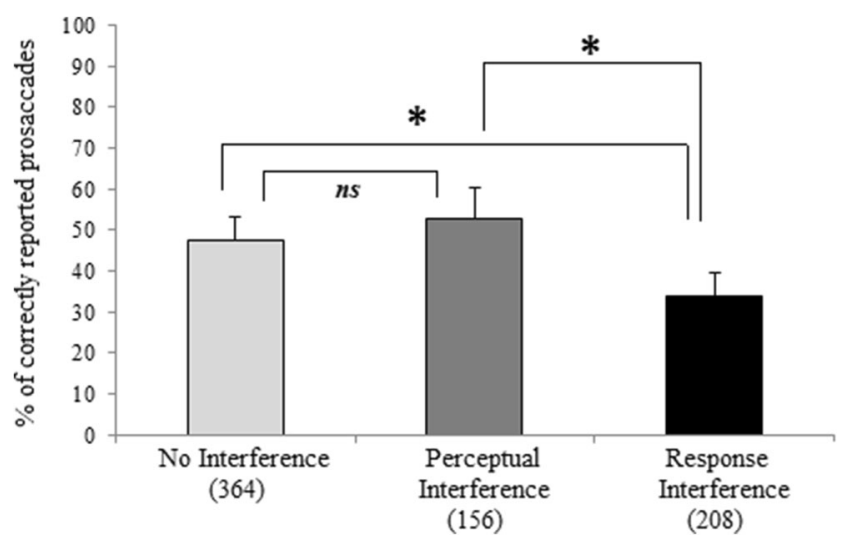

Fig. 8 Percentage of correctly detected erroneous prosaccades as a function of flanker condition $(*=$ statistically significant difference between conditions). Average number of trials per cell in each condition is reported in parentheses was likely driven by the fact that participants were less likely to make a corrective eye movement on RI than on NI and PI trials. Consequently, in Experiment 2, participants were more likely to report correctly on their initial eye movement simply by maintaining fixation at the final landing position of their first saccade. In Experiment 3, participants had to report explicitly on whether they thought they had made an error or not, rather than on the direction of their initial eye movement, and we found that this judgment was worse under conditions of high response interference. This is inconsistent with the hypothesis that general response interference aids conscious error detection.

\section{General discussion}

In these studies we examined how response interference affects people's awareness of their own errors in the antisaccade task. Before detailing how the manipulation of response interference in our paradigm may have led to the observed differences in self-report, we first review behavioral cross-task interactions between the antisaccade task and the two manual tasks.

First, we note that we replicated the classic patterns of performance on the mental rotation task and of response interference on both the flanker and antisaccade tasks. On the mental rotation task, we found that people were slower to judge the handedness of a letter, and made more errors, when the orientation of the letter was $180^{\circ}$ rather than $0^{\circ}, 90^{\circ}$, or $270^{\circ}$. On the flanker task, response interference was reflected in significantly slower reaction times on response incompatible relative to response compatible trials, and significantly lower accuracy scores on response incompatible relative to response compatible trials. On the antisaccade task, we found that out of all eye movements, approximately $6 \%$ were erroneous prosaccades. Out of these erroneous eye movements, approximately $50 \%$ were undetected by participants.

In addition to these task-specific effects of response interference, we found that participants' antisaccade latencies were slower on high-difficulty than on low-difficulty trials in the mental rotation task. On the flanker task we found that participants made a higher percentage of erroneous prosaccades on trials in which the flanker stimulus elicited response conflict. These results are consistent with previous evidence showing that some endogenous eye movements are dependent on an amodal response selection bottleneck (Pashler, Carrier, \& Hoffman, 1993). Neural evidence also suggests that both the antisaccade task and manual flanker task engage shared neural regions that are correlated with cognitive control and performance monitoring (Leung \& Cai, 2007). For instance, evidence from studies on the error-related negativity $(\mathrm{Ne})$ and error-related positivity (Pe), indicate that these ERP components are consistently evoked by both tasks (for review, see van Veen \& Carter, 2002). Thus, the fact we replicated 
behavioral cross-task interference in two different experiments is in line with prior neural data. Participants' tendency to make more erroneous prosaccades on flanker incompatible trials but not on high-difficulty mental rotation trials suggests that increased response competition, but not increased task difficulty alone, may perturb the initial programming of voluntary eye movements. The mental rotation task was undoubtedly difficult, as shown by the fact that mean mental rotation RT was approximately $200 \mathrm{~ms}$ longer than mean RT in the flanker task.

Although we found behavioral cross-task interactions, we did not find that either task difficulty or response interference improved participants' ability to consciously detect erroneous responses. Results from Experiment 1 showed that a correlate of response interference, that is, increased task difficulty, does not improve conscious error detection. In Experiment 2, we found that response interference appeared to improve participants' ability to consciously monitor their eye movements, but post hoc analyses indicated that this effect was driven by participants' tendency to make fewer corrective antisaccades on flanker incompatible trials. In Experiment 3, in which participants had to evaluate their enacted eye movements against an intended action plan, we no longer found that response interference improved error detection. In fact, in this experiment we found that participants' ability to monitor their eye movements was worse on response interference trials. As noted in the introduction to Experiment 1, the type of metacognitive judgment required of participants in Experiment 3 may induce an additional memory demand (e.g., maintenance of the intended action plan, and comparison of the enacted and intended action plan) that is not present if participants are simply asked to report on the action (i.e., saccade direction) that they produced. Increased response interference may interfere with some aspect of this metacognitive process.

Together, these findings help elucidate the role of response interference in error detection. According to one application of the conflict-monitoring model of error detection, conscious error perception depends on a single mechanism that takes input from activity generated by instances of response interference and the occurrence of erroneous responses (Yeung et al., 2004). Formally, response conflict is modeled as the product of two coactivated, incompatible response units (weighted by the strength of the inhibitory link between them), where greater values reflect increased response conflict. Presumably, if two tasks produce such signals simultaneously, this should lead to greater activation of the conflict monitoring mechanism, which may in turn increase error awareness. Therefore, under this account, response interference on the flanker task would be expected to amplify a signal that is used to determine the need for up-regulation of control on the antisaccade task. In turn, this increase in signal strength would contribute to participants' decision process when evaluating the outcome of their performance on the antisaccade task. Our data do not support such a link between general response interference and conscious error perception, however. One possible objection to our approach is that our dual-task paradigm manipulated response interference in one task (the flanker task) to see whether this would lead to an increase in error detection in a second task (the antisaccade task). We assumed that this would be the case if the conflictmonitoring model of error detection is correct because the flanker task and the antisaccade task both rely on an executive control network that operates in the resolution of conflict at the response selection stage of behavioral control (van Veen \& Carter, 2002). It is possible, however, that response signals from different tasks are encapsulated from one another, such that response interference from one task would not lead to increased error awareness in a second task.

Our results are also inconsistent with an alternative framework, according to which response interference may increase error detection by signaling an increased likelihood of error commission. According to this account, increased task difficulty should also lead to an increase in the conscious perception of errors. Support for this view comes from research showing that mental effort is positively correlated with tonic arousal (Howells, Stein, \& Russel, 2010) and some authors suggest that an increase in general physiological arousal might contribute to the detection of errors through global neural changes that modulate attention networks (Wessel et al., 2011). However, when we increased task demands by instructing participants to perform a difficult mental rotation task while executing a saccade we found no changes in error detection.

In sum, our study is the first to apply a dual-task design to systematically examine the relationship between error awareness and levels of response conflict and general task difficulty. When participants were required to simply monitor their actions (i.e., to indicate the direction of their initial eye movement, as in Experiment 2), we found that response interference improved action monitoring indirectly, that is, by reducing corrective behaviors. In contrast, when participants were asked to make a metacognitive judgment regarding whether they had committed an error or not (as in Experiment 3), self-report accuracy was worse on response interference than on response compatible trials. Although our results are inconsistent with the hypothesis that general response conflict improves awareness of errors, they are consistent with recent findings that increasing executive processing demands may perturb visual consciousness (De Loof, Poppe, Cleeremans, Gevers, \& Opstal, 2015) and metacognition (Maniscalco \& Lau, 2015). Maniscalco and Lau (2015) found that increased working-memory load selectively decreased metacognitive performance on a perceptual discrimination task, and they connected these findings to evidence that regions in the prefrontal cortex are integral to metacognitive processes (e.g., Henson, Rugg, Shallice, \& Dolan, 2000). Consistent with this 
evidence, we found that the flanker task, which engages prefrontal regions, interferes with participants' awareness of errors, whereas the mental rotation task, which has been shown to engage parietal regions (e.g., Harris et al., 2000), did not interfere with participants' self-monitoring. Although neuroimaging evidence suggests metacognition and cognitive control may engage distinct regions within the prefrontal cortex (e.g., Ridderinkhof, van den Wildenberg, Segalowitz, \& Carter, 2004), our behavioral results suggest that tasks that tax cognitive control may also disturb the awareness of one's own actions.

\section{References}

Beck, D. M., \& Lavie, N. (2005). Look here but ignore what you see: Effects of distractors at fixation. Journal of Experimental Psychology: Human Perception and Performance, 31, 592-607.

Blakemore, S. J., \& Frith, C. (2003). Self-awareness and action. Current Opinion in Neurobiology, 13, 219-224.

Bowling, A. C., Hindman, E. A., \& Donnelly, J. F. (2012). Prosaccade errors in the antisaccade task: Differences between corrected and uncorrected errors and links to neuropsychological tests. Experimental Brain Research, 216, 169-179.

Brown, J. W., \& Braver, T. S. (2005). Learned prediction of error likelihood in the anterior cingulate cortex. Science, 307, 1118-1121.

Cooper, L. A., \& Shepard, R. N. (1973). Chronometric studies of the rotation of mental images. In W. G. Chase (Ed.), Visual information processing (pp. 76-176). New York: Academic Press.

De Loof, E. D., Poppe, L., Cleeremans, A., Gevers, W., \& Opstal, F. V. (2015). Different effects of executive and visuopatial working memory on visual consciousness. Attention, Perception \& Psychophysics, 77, 2523-2528.

Desoto, M. C., Fabiani, M., Geary, D. C., \& Gratton, G. (2001). When in doubt, do it both ways: Brain evidence of the simultaneous activation of conflicting motor responses in a spatial Stroop task. Journal of Cognitive Neuroscience, 13, 523-536.

Eriksen, B. A., \& Eriksen, C. W. (1974). Effects of noise letters upon the identification of target letters in a non-search task. Perception \& Psychophysics, 16, 143-149.

Faul, F., Erdfelder, E., Lang, A.-G., \& Buchner, A. (2007). G*Power 3: A flexible statistical power analysis program for the social, behavioral, and biomedical sciences. Behavior Research Methods, 39, 175-191.

Godijn, R., \& Kramer, A. F. (2006). Prosaccades and antisaccades to onsets and color singletons: Evidence that erroneous prosaccades are not reflexive. Experimental Brain Research, 172, 439-448.

Hallett, P. E. (1978). Primary and secondary saccades to goals defined by instructions. Vision Research, 18, 1279-1296.

Hallett, P. E., \& Adams, B. D. (1980). The predictability of saccadic latency in a novel voluntary oculomotor task. Vision Research, 20, 329-339.

Harris, I. M., Egan, G. F., Sonkkila, C., Tochon-Danguy, H. J., Paxinos, G., \& Watson, J. D. G. (2000). Selective right parietal lobe activation during mental rotation: A parametric PET study. Brain, 123, 65-73.

Henson, R. N., Rugg, M. D., Shallice, T., \& Dolan, R. J. (2000). Confidence in recognition memory for words: Dissociating right prefrontal roles in episodic retrieval. Journal of Cognitive Neuroscience, 12, 913-923.

Howells, F. M., Stein, D. J., \& Russell, V. A. (2010). Perceived mental effort correlates with changes in tonic arousal during attentional tasks. Behavioral and Brain Functions. doi:10.1186/1744-9081-6-39
Hutton, S. B., \& Ettinger, U. (2006). The antisaccade task as a research tool in psychopathology: A critical review. Psychophysiology, 43, 302-313.

Irwin, D. E., \& Brockmole, J. R. (2000). Mental rotation is suppressed during saccadic eye movements. Psychonomic Bulletin \& Review, 7 , 654-661.

Irwin, D. E., \& Carlson-Radvansky, L. A. (1996). Cognitive suppression during saccadic eye movements. Psychological Science, 7, 83-88.

Klein, T. A., Ullsperger, M., \& Danielmeier, C. (2013). Error awareness and the insula: Links to neurological and psychiatric diseases. Frontiers in Human Neuroscience, 7, 14.

Kristjansson, A., Chen, Y., \& Nakayama, K. (2001). Less attention is more in the preparation of antisaccades, but not prosaccades. Nature Neuroscience, 4, 1037-1042.

Leung, H. C., \& Cai, W. (2007). Common and differential ventrolateral prefrontal activity during inhibition of hand and eye movements. The Journal of Neuroscience, 27, 9893-9900.

Lindsay, D. S., \& Jacoby, L. L. (1994). Stroop process dissociations: The relationship between facilitation and interference. Journal of Experimental Psychology, 20, 219-234.

Logan, G. D., \& Zbrodoff, N. J. (1979). When it helps to be misled: Facilitative effects of increasing the frequency of conflicting stimuli in a Stroop-like task. Memory and Cognition, 7, 166-174.

MacLeod, C. M. (1991). Half a century of research on the Stroop effect: An integrative review. Psychological Bulletin, 109, 163-203.

Maniscalco, B., \& Lau, H. (2015). Manipulation of working memory contents selectively impairs metacognitive sensitivity in a concurrent visual discrimination task. Neuroscience of Consciousness, 113. doi:10.1093/nc/niv002

Mokler, A., \& Fischer, B. (1999). The recognition and correction of involuntary prosaccades in an antisaccade task. Experimental Brain Research, 125, 511-516.

Nee, D. E., Wager, T. D., \& Jonides, J. (2007). Interference resolution: Insights from a meta-analysis of neuroimaging tasks. Cognitive, Affective, and Behavioral Neuroscience, 7, 1-17.

Pashler, H., Carrier, M., \& Hoffman, J. (1993). Saccadic eye movements and dual-task interference. The Quarterly Journal of Experimental Psychology, 46, 51-82.

Peterburs, J., Pergola, G., Koch, B., Schwarz, Hoffman, K. P., Daum, I., \& Bellebaum, C. (2011). Altered error processing following vascular thalamic damage: Evidence from an antisaccade task. PLOS ONE, 6, e21517. doi:10.1371/journal.pone.0021517

Rabbitt, P. M. A. (1968). Three kinds of error-signalling responses in a serial choice task. Quarterly Journal of Experimental Psychology, 20, 179-188.

Ridderinkhof, K. R., van den Wildenberg, W. P., Segalowitz, S. J., \& Carter, C. S. (2004). Neurocognitive mechanisms of cognitive control: The role of prefrontal cortex in action selection, response inhibition, performance monitoring, and reward-based learning. Brain \& Cognition, 56, 129-140.

Rouder, J. N., Speckman, P. L., Sun, D., Morey, R. D., \& Iverson, G. (2009). Bayesian $t$ tests for accepting and rejecting the null hypothesis. Psychonomic Bulletin \& Review, 16, 225-237.

Simon, J. R. (1969). Reactions towards the source of stimulation. Journal of Experimental Psychology, 81, 174-176.

Steinhauser, M., Maier, M., \& Hubner, R. (2008). Modeling behavioral measures of error detection in choice tasks: Response monitoring versus conflict monitoring. Journal of Experimental Psychology: Human Perception and Performance, 34, 158-176.

Taylor, A., \& Hutton, S. (2011). Error awareness and antisaccade performance. Experimental Brain Research, 213, 27-34.

Ullsperger, M., Harsay, H. A., Wessel, J. R., \& Ridderinkhof, K. R. (2010). Conscious perception of errors and its relation to the anterior insula. Brain Structure and Function, 214, 629-643.

Unsworth, N., Schrock, J., \& Engle, R. (2004). Working memory capacity and the antisaccade task: Individual differences in voluntary 
saccade control. Journal of Experimental Psychology: Learning, Memory, \& Cognition, 30, 1302-1321.

van Veen, V., \& Carter, C. S. (2002). The timing of action monitoring in rostral and caudal anterior cingulate cortex. Journal of Cognitive Neuroscience, 14, 593-602.

Wessel, J. R. (2012). Error awareness and the error-related negativity: Evaluating the first decade of evidence. Frontiers in Human Neuroscience, 6, 88.
Wessel, J. R., Danielmeier, C., \& Ullsperger, M. (2011). Error awareness revisited: The ERN, the Pe, and the autonomous nervous system. Journal of Cognitive Neuroscience, 23, 3021-3036.

Yeung, N., Botvinick, M. M., \& Cohen, J. D. (2004). The neural basis of error detection: Conflict monitoring and the error-related negativity. Psychological Review, 111, 931-959. 